\title{
Reversal of the Methylation-Associated Regulation of miR-200a/b by Helicobacter pylori Eradication Contributes to the Chemoprevention of Gastric Carcinogenesis
}

\author{
Nayoung Kim ${ }^{1,2}$ \\ ${ }^{1}$ Department of Internal Medicine, Seoul National University Bundang Hospital, Seongnam, and ${ }^{2}$ Department of Internal Medicine and Liver \\ Research Institute, Seoul National University College of Medicine, Seoul, Korea
}

See "Helicobacter pylori Eradication Can Reverse the Methylation-Associated Regulation of miR-200a/b in Gastric Carcinogenesis" by Ji Min Choi, et al. on page 571, Vol. 14, No. 5, 2020

MicroRNAs (miRNAs) are currently considered as crucial post-transcriptional regulators of gene expression. ${ }^{1}$ Their roles in development, cell proliferation and differentiation are widely recognized. Furthermore, miRNAs are frequently altered in cancer cells and reveal their functions as either oncogenes or tumor suppressors. ${ }^{1}$ The emerging role of miRNAs in diverse and fundamental cellular mechanisms suggests that proper control of these regulatory elements is essential for the maintenance of a nonpathological state. ${ }^{1}$ Recent reports have highlighted the regulatory role of miRNAs in Helicobacter pylori infection and associated diseases. ${ }^{2}$ For example, a strong inflammatory response characterized by the early production of proinflammatory tumor necrosis factor (TNF)- $\alpha$ and interleukin (IL)-6 cytokines, followed by IL-10, IL-1 $\beta$ and IL-23 secretion as a consequence of miR-146a up-regulation and strong miR-155 induction, which raised the TNF- $\alpha$ production. ${ }^{2}$ In contrast, IL8 , TNF- $\alpha$ and IL- $\beta$ could contribute to the induction of miR146a during $H$. pylori infection. ${ }^{2}$ Therefore, miRNAs modulate the $H$. pylori infection and are also affected by these bacteria, as, for example, the synthesis of the transcription factor nuclear factor- $\kappa \mathrm{B}$ that can act as a transactivator of miR-200b and miR200c. ${ }^{2}$ Similarly, epigenetic change is one of the mechanisms that regulates the expression of miRNAs and has been known to play a role in $H$. pylori-associated gastric carcinogenesis. ${ }^{3,4}$ From this background Choi et al. ${ }^{5}$ aimed to evaluate the epigenetic changes of miR-200a/b in $H$. pylori-associated gastric carcinogenesis and whether $H$. pylori eradication restore epigenetic changes of miR-200a/b. The miR-200 family is closely linked to the expression of ZEB1 and ZEB2, key regulators of epithelialmesenchymal transition (EMT), and regulates crucial processes in carcinogenesis, such as tumor initiation, progression, invasion, and metastasis of various types of cancer. ${ }^{5}$ Recent studies have shown that miR-200 family were downregulated in gastric cancer (GC), suggesting its role as a tumor suppressor in $\mathrm{GC}^{6}$ In addition, hypermethylation of the promoter $\mathrm{CpG}$ island was one of the mechanisms of miR-200c/141 downregulation. ${ }^{6}$ However, it has not yet been fully elucidated whether epigenetic alterations in the miR-200 family are affected by $H$. pylori infection and/or by its eradication. Choi et al. ${ }^{5}$ evaluated the expression and methylation levels of miR-200a/b in GC cell lines, human gastric mucosa of $H$. pylori-negative and -positive controls, and H. pyloripositive GC patients. They found that the level of miR200a/b methylation decreased and the level of expression increased after demethylation. ${ }^{5}$ Furthermore, the miR-200a/b methylation levels in the human gastric mucosa, increased in the order from $H$. pylori-negative control group, H. pylori-positive control group, and H. pylori-positive GC group. Interestingly the level of methylation decreased and the level of expression of miR-200a/b increased significantly only after 6 months in the $H$. pylori-eradicated group. ${ }^{5}$ These results suggest that epigenetic alterations of miR200a/b may be implicated in H. pylori-induced gastric carcinogenesis and $H$. pylori eradication improved the field defect for cancerization.

The causal association between $H$. pylori infection and GC has been firmly established by many epidemiological and clinical studies. Although GC develops in approximately 1\% to 3\%

\footnotetext{
Correspondence to: Nayoung Kim

Department of Internal Medicine, Seoul National University Bundang Hospital, 82 Gumi-ro 173beon-gil, Bundang-gu, Seongnam 13620, Korea

Tel: +82-31-787-7008, Fax: +82-31-787-4051, E-mail: nayoungkim49@empas.com, nakim49@snu.ac.kr pISSN 1976-2283 eISSN 2005-1212 https://doi.org/10.5009/gnl20251

(c) This is an Open Access article distributed under the terms of the Creative Commons Attribution Non-Commercial License (http://creativecommons.org/licenses/by-nc/4.0) which permits unrestricted non-commercial use, distribution, and reproduction in any medium, provided the original work is properly cited.
} 
of $H$. pylori-infected subjects, more than 90\% of GC patients have a current or past $H$. pylori infection. ${ }^{7}$ In addition, a metaanalysis of 11 case-control studies including larger subjects also reported a strong association between $H$. pylori infection and GC (odds ratio for GC in H. pylori-infected subjects, 3.00; 95\% confidence interval [CI], 2.42 to 3.72). ${ }^{8}$ In addition, many studies have demonstrated the beneficial effect of $H$. pylori eradication by reduction of the incidence risk of GC. A meta-analysis of seven randomized controlled trials regarding the effectiveness of $H$. pylori eradication in the prevention of primary GC in healthy asymptomatic individuals showed that overall risk ratio and risk difference were 0.67 (95\% CI, 0.48 to 0.95 ) and -0.00 (95\% CI, -0.01 to 0.00). ${ }^{9}$ Recently Choi et al. ${ }^{10}$ nicely showed the chemopreventive effect of $H$. pylori eradication in the first GC family over 9.2 years follow-up in 1,676 participants. Our team also reported the chemopreventive effect of $H$. pylori eradication after multivariable adjustment in a comprehensive manner. ${ }^{7}$ However, it remains unclear how $H$. pylori eradication prevents GC. Our team proposed that its underlying mechanism includes reversibility of atrophic gastritis and intestinal metaplasia, methylation, EMT, and stem cells. ${ }^{7}$ However, it has not yet been established whether EMT of non-neoplastic gastric epithelial cells is directly associated with $H$. pylori infection. ${ }^{7}$ Now Choi et al. proposed the methylation of miR-200a/b, which is involved in EMT as one of mechanism of $H$. pylori-induced gastric carcinogenesis. $^{5}$ As $H$. pylori eradication improved the field defect for cancerization by methylation of $\mathrm{miR}-200 \mathrm{a} / \mathrm{b}^{5}$ it might be added as one of mechanisms how $H$. pylori eradication prevents GC. In 2018 Korean Ministry of Health and Welfare extended indications of $H$. pylori eradication including to the conditions when doctors think $H$. pylori eradication is helpful for individuals. Maybe in the future the insurance could be extended into all of $H$. pylori-associated gastritis like in Japan in 2013 because our country has the infamy that the incidence of GC is the first in the world so far.

\section{CONFLICTS OF INTEREST}

No potential conflict of interest relevant to this article was reported.

\section{ORCID}

Nayoung Kim

https://orcid.org/0000-0002-9397-0406

\section{REFERENCES}

1. Belair C, Darfeuille F, Staedel C. Helicobacter pylori and gastric cancer: possible role of microRNAs in this intimate relationship. Clin Microbiol Infect 2009;15:806-812.

2. Cadamuro AC, Rossi AF, Maniezzo NM, Silva AE. Helicobacter pylori infection: host immune response, implications on gene expression and microRNAs. World J Gastroenterol 2014;20:14241437.

3. Noto JM, Peek RM. The role of microRNAs in Helicobacter pylori pathogenesis and gastric carcinogenesis. Front Cell Infect Microbiol 2012;1:21.

4. Zabaleta J. MicroRNA: a bridge from H. pylori infection to gastritis and gastric cancer development. Front Genet 2012;3:294.

5. Choi JM, Kim SG, Yang HJ, et al. Helicobacter pylori eradication can reverse the methylation-associated regulation of miR-200a/b in gastric carcinogenesis. Gut Liver 2020;14:571-580.

6. Zhou X, Wang Y, Shan B, et al. The downregulation of miR200c/141 promotes ZEB1/2 expression and gastric cancer progression. Med Oncol 2015;32:428.

7. Kim N. Chemoprevention of gastric cancer by Helicobacter pylori eradication and its underlying mechanism. J Gastroenterol Hepatol 2019;34:1287-1295.

8. Xue FB, Xu YY, Wan Y, Pan BR, Ren J, Fan DM. Association of H. pylori infection with gastric carcinoma: a meta analysis. World $J$ Gastroenterol 2001;7:801-804.

9. Seta T, Takahashi Y, Noguchi Y, et al. Effectiveness of Helicobacter pylori eradication in the prevention of primary gastric cancer in healthy asymptomatic people: a systematic review and meta-analysis comparing risk ratio with risk difference. PLoS One 2017;12:e0183321.

10. Choi IJ, Kim CG, Lee JY, et al. Family history of gastric cancer and Helicobacter pylori treatment. N Engl J Med 2020;382:427-436. 